\title{
Precoded Faster-than-Nyquist Signaling with Optimal Power Allocation in Frequency-Selective Channel
}

\author{
Takumi Ishihara and Shinya Sugiura* \\ Institute of Industrial Science, The University of Tokyo \\ Meguro-Ku, Tokyo 153-8505, Japan \\ Email: \{t.ishihara@ieee.org, sugiura@iis.u-tokyo.ac.jp\}
}

\begin{abstract}
In this paper, we propose eigendecompositionprecoded faster-than-Nyquist (FTN) signaling with power allocation in a frequency-selective fading channel. More specifically, we derive mutual information associated with the proposed FTN signaling. Then, the optimal power coefficients are calculated such that the derived mutual information is maximized. Our analytical performance results show that the proposed FTN signaling scheme achieves a higher information rate than the conventional FTN signaling scheme without relying on power allocation and the classic Nyquist-based signaling scheme, under the assumption that all the schemes employ a root-raised cosine shaping filter. Moreover, our numerical simulation results of the bit error ratio performance and the power spectral density demonstrate that the proposed FTN scheme outperforms the conventional Nyquist-based signaling scheme without sacrificing any bandwidth broadening.

Index Terms-Faster-than-Nyquist signaling, Nyquist criterion, eigenvalue decomposition, frequency-selective channel, information-theoretic analysis, capacity, information rate, power allocation.
\end{abstract}

\section{INTRODUCTION}

The classic Nyquist criterion has played an essential role in bandlimited communication systems, having finite bandwidth $2 W \mathrm{~Hz}$, since it achieves intersymbol interference (ISI)-free information transmission. In the Nyquist criterion, a minimum symbol interval is limited to $T_{0}=1 /(2 W)$ [s], and hence the achievable symbol rate is upper-bounded by $1 / T_{0}$. To overcome this limitation, the concept of faster-than-Nyquist (FTN) signaling has been studied $[1,2]$. In FTN signaling, a symbol interval is defined by $T=\tau T_{0}(0<\tau \leq 1)$, where $\tau$ is a symbol's packing ratio. Therefore, FTN signaling has the potential of achieving a higher transmission rate than the conventional Nyquist-based ISI-free counterpart without imposing any extra bandwidth. Several properties of FTN signaling have been revealed in the literature [1,3-10]. In [1], it was shown that the minimum Euclidean distance (MED) of FTN signaling is the same as that of Nyquist-based signaling for $\tau \geq 0.802$ under the assumption of the ideal

Postprint accepted on 24 March 2021 for publication in IEEE International Conference on Communications Workshops (IEEE ICC Workshops), 2021. (C) 2021 IEEE. Personal use of this material is permitted. Permission from IEEE must be obtained for all other uses, in any current or future media, including reprinting/republishing this material for advertising or promotional purposes, creating new collective works, for resale or redistribution to servers or lists, or reuse of any copyrighted component of this work in other works. rectangular shaping filter (sinc pulse). In [3], the capacity of FTN signaling was derived for the first time, where the use of a root raised-cosine (RRC) shaping filter having a roll-off factor $\beta$ was assumed. It was revealed that FTN signaling achieves a higher capacity than the conventional Nyquist-based counterpart employing the same RRC shaping filter, owing to the exploitation of the excess bandwidth. In [5], an achievable information rate of FTN signaling for a finite block-length was analyzed.

Furthermore, information-theoretic analysis of precoded FTN signaling was carried out based on the matrix factorization [7-10]. In [7], the capacity of eigenvalue decomposition (EVD)-precoded FTN signaling was derived based on the eigenvalue distribution analysis of an FTN-induced ISI matrix. The capacity derived in [7] coincided with that of [3]. In [8], power allocation was introduced in EVD-precoded FTN signaling, where the power allocation coefficients were optimized such that the mutual information of EVD-precoded FTN signaling is maximized, based on the Lagrange multiplier method. The EVD-precoded FTN signaling scheme with optimal power allocation achieves a higher capacity than unprecoded FTN signaling and Nyquist-based signaling counterparts. However, the FTN-induced ISI matrix typically includes significantly low eigenvalues for a small packing ratio in the range of $\tau<1 /(1+\beta)$, while optimal power allocation proposed in [8] made an idealistic assumption that such very low eigenvalues are precisely computed. Since it is challenging to compute significantly low eigenvalues accurately, truncated power allocation was further proposed in [8], where substreams associated with significantly low eigenvalues were deactivated. Moreover, in [9], it was revealed that in EVD-precoded FTN signaling with power allocation, there exists a tradeoff between the achievable information rate and the calculation precision of the floating-point arithmetic employed. More specifically, higher calculation precision leads to a higher information rate since the number of activated substreams increases. However, in the previous informationtheoretic studies [7-11], the simplified additive white Gaussian noise (AWGN) and frequency-flat channels were assumed, and a more realistic frequency-selective fading channel has not been considered.

The novel contributions of this paper are as follows. Moti- 
vated by the conventional precoded FTN signaling [8], EVDprecoded FTN signaling with power allocation is proposed in the scenario of a frequency-selective fading channel. More specifically, we derive mutual information of FTN signaling for a frequency-selective fading channel, and then the design guideline of the optimal power coefficients are provided such that the derived mutual information is maximized. Analytical performance results show that the proposed FTN signaling scheme achieves a higher information rate than the FTN signaling scheme without relying on power allocation and the classic Nyquist-based signaling scheme under the assumption that the same RRC shaping filter is employed in each scheme. More specifically, the proposed FTN scheme achieves the information rate close to the performance upper bound of Nyquist signaling with an idealistic rectangular shaping filter while employing a practical RRC shaping filter.

\section{Proposed FTN Signaling With Power Allocation in Frequency-Selective ChanNel}

\section{A. System Model of Linearly-Precoded FTN Signaling}

First, we introduce the system model of linearly-precoded FTN signaling for the frequency-selective fading channel. At the transmitter, information bits are modulated onto $N$ length complex-valued symbols $\mathbf{s}=\left[s_{0}, \cdots, s_{N-1}\right]^{T} \in \mathbb{C}^{N}$, which has $\mathbb{E}\left[\left|s_{n}\right|^{2}\right]=\sigma_{s}^{2}(n=0, \cdots, N-1)$, where $\mathbb{E}[\cdot]$ denotes the expectation operation. Note that we consider the block transmission and the effects of inter-block interference (IBI) are ignored similar to most previous FTN studies. Then, the modulated symbols $\mathbf{s}$ are precoded by a linear-precoding matrix of $\mathbf{P} \in \mathbb{C}^{N \times N}$ as follows:

$$
\begin{aligned}
\mathbf{x} & =\left[x_{0}, \cdots, x_{N-1}\right]^{T} \in \mathbb{C}^{N} \\
& =\text { Ps. }
\end{aligned}
$$

The precoded symbols $\mathbf{x}$ are passed through the RRC shaping filter $h(t)$ having a roll-off factor $\beta$ and a unit energy of $\int_{-\infty}^{\infty}|h(t)|^{2} d t=1$. Then, the bandlimited signal is transmitted with an FTN-specific symbol interval $T=\tau T_{0}$. The transmit FTN signal $x(t)$ is expressed by

$$
x(t)=\sum_{n} x_{n} h(t-n T) .
$$

In an $L$-tap frequency-selective fading channel, the received FTN signal after passing through a matched filter $h^{*}(-t)$ at the receiver is given by [12-15]

$$
r(t)=\sum_{l=0}^{L-1} \sum_{n} h_{l} x_{n} g(t-(l+n) T)+\eta(t),
$$

where

$$
\begin{aligned}
g(t) & =\int_{-\infty}^{\infty} h(\zeta) h^{*}(\zeta-t) d \zeta \\
\eta(t) & =\int_{-\infty}^{\infty} n(\zeta) h^{*}(\zeta-t) d \zeta .
\end{aligned}
$$

Here, $n(t)$ is a complex-valued AWGN, represented by a complex-valued white Gaussian random process with a zero mean and a spectral density of $N_{0}$. Furthermore, $h_{l}(l=$ $0, \cdots, L-1)$ represent the channel coefficients.

Moreover, by defining the $k$ th received sample $r_{k}=$ $r(k T) \quad(k=0, \cdots, N-1)$, the received sample block is expressed by

$$
\begin{aligned}
\mathbf{r} & =\left[r_{0}, \cdots, r_{N-1}\right]^{T} \in \mathbb{C}^{N} \\
& =\mathbf{G}_{h} \mathbf{x}+\boldsymbol{\eta},
\end{aligned}
$$

where the noise components $\boldsymbol{\eta}=[\eta(0), \cdots, \eta((N-1) T)]^{T}$ have an FTN-specific correlation of $\mathbb{E}\left[\boldsymbol{\eta} \boldsymbol{\eta}^{H}\right]=N_{0} \mathbf{G}$ [3], and $\mathbf{G} \in \mathbb{R}^{N \times N}$ is a Toeplitz matrix whose first column is given by $[g(0), g(T), \cdots, g((N-1) T)]^{T}$. Additionally, the $k$ th-row and $m$ th-column element of the ISI matrix $\mathbf{G}_{h} \in \mathbb{C}^{N \times N}$, which includes both the effects of the FTN-induced ISI and the dispersive channel, is given by

$$
\mathbf{G}_{h}(k, m)=\sum_{l=0}^{L-1} h_{l} g(k T-(m+l) T) .
$$

The weight matrix $\mathbf{W}$ is multiplied by the received block $\mathbf{r}$ of (8) as follows:

$$
\begin{aligned}
\mathbf{r}_{\mathrm{d}} & =\mathbf{W r} \\
& =\mathbf{W G}_{h} \mathbf{x}+\mathbf{W} \boldsymbol{\eta} .
\end{aligned}
$$

The average transmit energy per block is calculated by [8]

$$
\begin{aligned}
E_{N} & =\mathbb{E}\left[\int_{-\infty}^{\infty}|x(t)|^{2} d t\right] \\
& =\mathbb{E}\left[\sum_{k} \sum_{m} x_{k} x_{m}^{*} g((k-m) T)\right] \\
& =\mathbb{E}\left[\mathbf{x}^{H} \mathbf{G x}\right] \\
& =\mathbb{E}\left[\mathbf{s}^{H} \mathbf{P}^{H} \mathbf{G P s}\right] .
\end{aligned}
$$

If $\mathbf{P}=\mathbf{I}_{N}$, the average transmit energy becomes $E_{N}=N \sigma_{s}^{2}$, where $\mathbf{I}_{N}$ is the $(N \times N)$-sized identity matrix [8]. Hence, the precoding matrix $\mathbf{P}$ has to be designed such that $E_{N}=N \sigma_{s}^{2}$ is satisfied. Note that based on EVD, the FTN-induced ISI matrix $\mathbf{G}$ is factorized into $[7,16]$

$$
\mathbf{G}=\mathbf{V} \mathbf{\Lambda} \mathbf{V}^{T}
$$

where $\mathbf{V} \in \mathbb{R}^{N \times N}$ is an orthogonal matrix. Moreover, $\boldsymbol{\Lambda} \in \mathbb{R}^{N \times N}$ is a diagonal matrix having the diagonal entry of $\lambda_{0}, \cdots, \lambda_{N-1}$, i.e., $\boldsymbol{\Lambda}=\operatorname{diag}\left[\lambda_{0}, \cdots, \lambda_{N-1}\right]$, which are in descending order.

\section{B. Mutual Information}

Mutual information between the received block $\mathbf{r}$ and the transmit symbols $\mathrm{x}$ is calculated by

$$
\begin{aligned}
I(\mathbf{x} ; \mathbf{r}) & =h_{e}(\mathbf{r})-h_{e}(\mathbf{r} \mid \mathbf{x}) \\
& =h_{e}(\mathbf{r})-h_{e}(\boldsymbol{\eta}),
\end{aligned}
$$

where $h_{e}(\cdot)$ represents a differential entropy. The upper bounds of the differential entropies of $\mathbf{r}$ and $\boldsymbol{\eta}$ are given by $h_{e}(\mathbf{r}) \leq \log _{2}\left((\pi e)^{N}\left|\mathbb{E}\left[\mathbf{r r}^{H}\right]\right|_{\text {det }}\right)$ and $h_{e}(\boldsymbol{\eta}) \leq$ $\log _{2}\left((\pi e)^{N}\left|\mathbb{E}\left[\boldsymbol{\eta} \boldsymbol{\eta}^{H}\right]\right|_{\text {det }}\right)$ [17], where $|\cdot|_{\text {det }}$ denotes the 
determinant operation. For an instantaneous fading channel, the covariance matrix $\mathbb{E}\left[\mathbf{r r}^{H}\right]$ is given by

$$
\begin{aligned}
\mathbb{E}\left[\mathbf{r r}^{H}\right] & =\mathbb{E}\left[\left(\mathbf{G}_{h} \mathbf{x}+\boldsymbol{\eta}\right)\left(\mathbf{G}_{h} \mathbf{x}+\boldsymbol{\eta}\right)^{H}\right] \\
& =\mathbf{G}_{h} \mathbf{R}_{x} \mathbf{G}_{h}^{H}+N_{0} \mathbf{G}
\end{aligned}
$$

where we have $\mathbf{R}_{x}=\mathbb{E}\left[\mathbf{x} \mathbf{x}^{H}\right]$. Hence, the mutual information $I(\mathbf{x} ; \mathbf{r})$ is upper-bounded as follows:

$$
\begin{aligned}
I(\mathbf{x} ; \mathbf{r}) & \leq \log _{2} \frac{\left|\mathbf{G}_{h} \mathbf{R}_{x} \mathbf{G}_{h}^{H}+N_{0} \mathbf{G}\right|_{\mathrm{det}}}{\left|N_{0} \mathbf{G}\right|_{\operatorname{det}}} \\
& =\log _{2}\left|\mathbf{I}_{N}+\frac{1}{N_{0}} \mathbf{G}_{h} \mathbf{R}_{x} \mathbf{G}_{h}^{H} \mathbf{G}^{-1}\right|_{\mathrm{det}},
\end{aligned}
$$

where the relationship of $|\mathbf{A}|_{\text {det }}^{-1}=\left|\mathbf{A}^{-1}\right|_{\text {det }}$ is used. Furthermore, since $\mathbf{G}$ is positive-definite for the finite block length $N$ [7], we have the relationship of

$$
\begin{aligned}
\mathbf{G}^{-1} & =\mathbf{V} \boldsymbol{\Lambda}^{-1} \mathbf{V}^{T} \\
& =\mathbf{V} \boldsymbol{\Lambda}^{-\frac{1}{2}} \boldsymbol{\Lambda}^{-\frac{1}{2}} \mathbf{V}^{T} .
\end{aligned}
$$

With (24), (22) is modified to

$$
\begin{aligned}
I(\mathbf{x} ; \mathbf{r}) & \leq \log _{2}\left|\mathbf{I}_{N}+\frac{1}{N_{0}} \mathbf{G}_{h} \mathbf{R}_{x} \mathbf{G}_{h}^{H} \mathbf{V} \boldsymbol{\Lambda}^{-1} \mathbf{V}^{T}\right|_{\mathrm{det}} \\
& =\log _{2}\left|\mathbf{I}_{N}+\frac{1}{N_{0}} \mathbf{R}_{x} \mathbf{G}_{h}^{H} \mathbf{V} \boldsymbol{\Lambda}^{-\frac{1}{2}} \boldsymbol{\Lambda}^{-\frac{1}{2}} \mathbf{V}^{T} \mathbf{G}_{h}\right|_{\mathrm{det}} \\
& =\log _{2}\left|\mathbf{I}_{N}+\frac{1}{N_{0}} \mathbf{R}_{x} \mathbf{D}^{H} \mathbf{D}\right|_{\mathrm{det}}
\end{aligned}
$$

where

$$
\mathbf{D}=\boldsymbol{\Lambda}^{-\frac{1}{2}} \mathbf{V}^{T} \mathbf{G}_{h}
$$

Note that the relationship of $\left|\mathbf{I}_{N}+\mathbf{A B}\right|_{\text {det }}=\left|\mathbf{I}_{N}+\mathbf{B A}\right|_{\text {det }}$ is used for the modification from (25) to (26). Based on EVD, the matrix $\mathbf{D}^{H} \mathbf{D}$ in (27) is factorized into $\mathbf{D}^{H} \mathbf{D}=\mathbf{U} \boldsymbol{\Xi} \mathbf{U}^{H}$, where $\mathbf{U} \in \mathbb{C}^{N \times N}$ is an orthogonal matrix, and $\boldsymbol{\Xi} \in \mathbb{R}^{N \times N}$ is a diagonal matrix having $N$ descending-order eigenvalues of $\xi_{0}, \cdots, \xi_{N-1}$. Hence, (27) is further modified to

$$
\begin{aligned}
I(\mathbf{x} ; \mathbf{r}) & \leq \log _{2}\left|\mathbf{I}_{N}+\frac{1}{N_{0}} \mathbf{R}_{x} \mathbf{U} \boldsymbol{\Xi} \mathbf{U}^{H}\right|_{\mathrm{det}} \\
& =\log _{2}\left|\mathbf{I}_{N}+\frac{1}{N_{0}} \boldsymbol{\Xi}^{\frac{1}{2}} \mathbf{U}^{H} \mathbf{R}_{x} \mathbf{U} \boldsymbol{\Xi}^{\frac{1}{2}}\right|_{\mathrm{det}} .
\end{aligned}
$$

Note that the matrix of $\boldsymbol{\Xi}^{\frac{1}{2}} \mathbf{U}^{H} \mathbf{R}_{x} \mathbf{U} \boldsymbol{\Xi}^{\frac{1}{2}}=$ $\left(\mathbf{R}_{x}^{\frac{1}{2}} \mathbf{U} \boldsymbol{\Xi}^{\frac{1}{2}}\right)^{H} \mathbf{R}_{x}^{\frac{1}{2}} \mathbf{U} \boldsymbol{\Xi}^{\frac{1}{2}}$ in (30) is a positive semi-definite Hermitian matrix.

According to Hadamard's inequality, the determinant of an $(N \times N)$-sized positive semi-definite Hermitian matrix $\mathbf{C}$ is upper-bounded by $|\mathbf{C}|_{\text {det }} \leq \prod_{k=0}^{N-1} \mathbf{C}(k, k)[17,18]$, where $\mathbf{C}(k, k)$ denotes the $k$ th diagonal entry of $\mathbf{C}$. The equality holds if and only if $\mathbf{C}$ is a diagonal matrix. Hence, the mutual information (30) is maximized when $\boldsymbol{\Xi}^{\frac{1}{2}} \mathbf{U}^{H} \mathbf{R}_{x} \mathbf{U} \boldsymbol{\Xi}^{\frac{1}{2}}$ becomes a diagonal matrix, i.e., when $\mathbf{U}^{H} \mathbf{R}_{x} \mathbf{U}$ becomes a diagonal matrix. To satisfy this diagonal relationship while maintaining the power constraint, let us consider

$$
\begin{aligned}
\mathbf{U}^{H} \mathbf{R}_{x} \mathbf{U} & =\sigma_{s}^{2} \boldsymbol{\Gamma} \\
\mathbf{R}_{x} & =\sigma_{s}^{2} \mathbf{U} \boldsymbol{\Gamma} \mathbf{U}^{H},
\end{aligned}
$$

where we have a real-valued diagonal matrix of

$$
\boldsymbol{\Gamma}=\operatorname{diag}\left[\gamma_{0}, \cdots, \gamma_{N-1}\right] \in \mathbb{R}^{N \times N} .
$$

Here, (32) is achievable when we set the precoding matrix as

$$
\mathbf{P}=\mathbf{U} \boldsymbol{\Gamma}^{\frac{1}{2}}
$$

which provides the precoded symbols of $\mathbf{x}=\mathbf{U} \boldsymbol{\Gamma}^{\frac{1}{2}} \mathbf{s}$. With (32), the mutual information (30) is upper-bounded by

$$
\begin{aligned}
I(\mathbf{x} ; \mathbf{y}) & \leq \log _{2}\left|\mathbf{I}_{N}+\frac{\sigma_{s}^{2}}{N_{0}} \boldsymbol{\Xi}^{\frac{1}{2}} \boldsymbol{\Gamma} \boldsymbol{\Xi}^{\frac{1}{2}}\right|_{\mathrm{det}} \\
& =\sum_{k=0}^{N-1} \log _{2}\left(1+\frac{\sigma_{s}^{2}}{N_{0}} \gamma_{k} \xi_{k}\right) .
\end{aligned}
$$

Note that $\Gamma$ has to satisfy the energy constraint of $E_{N}=$ $N \sigma_{s}^{2}$. More specifically, From (14), the transmit energy per block for the precoded symbols of $\mathbf{x}=\mathbf{U} \boldsymbol{\Gamma}^{\frac{1}{2}} \mathbf{S}$ is calculated by

$$
\begin{aligned}
E_{N} & =\mathbb{E}\left[\mathbf{x}^{H} \mathbf{G} \mathbf{x}\right] \\
& =\mathbb{E}\left[\operatorname{tr}\left\{\mathbf{x}^{H} \mathbf{G} \mathbf{x}\right\}\right] \\
& =\mathbb{E}\left[\operatorname{tr}\left\{\mathbf{G} \mathbf{x} \mathbf{x}^{H}\right\}\right] \\
& =\operatorname{tr}\left\{\mathbf{G} \mathbf{U} \boldsymbol{\Gamma}^{\frac{1}{2}} \mathbb{E}\left[\mathbf{s s}^{H}\right] \boldsymbol{\Gamma}^{\frac{1}{2}} \mathbf{U}^{H}\right\} \\
& =\sigma_{s}^{2} \operatorname{tr}\{\boldsymbol{\Gamma} \mathbf{\Phi}\} \\
& =\sigma_{s}^{2} \sum_{k=0}^{N-1} \gamma_{k} \phi_{k},
\end{aligned}
$$

where $\boldsymbol{\Phi}=\mathbf{U}^{H} \mathbf{G} \mathbf{U}$, and $\phi_{k}$ represents the $k$ th diagonal element of $\boldsymbol{\Phi}$. Also, $\operatorname{tr}\{\cdot\}$ represents the trace operation. Hence, with the relationship of $E_{N}=N \sigma_{s}^{2}$ and (42), we have the energy constraint of

$$
\sum_{k=0}^{N-1} \gamma_{k} \phi_{k}=N
$$

\section{Optimal Power Allocation}

In this section, we derive the optimal coefficients $\gamma_{k}$ that maximize the mutual information (36), based on the Lagrange multiplier method.

The Lagrange function is formulated as follows:

$$
J=\sum_{k=0}^{N-1} \log _{2}\left(1+\frac{\sigma_{s}^{2}}{N_{0}} \gamma_{k} \xi_{k}\right)-\alpha\left(\sum_{k=0}^{N-1} \gamma_{k} \phi_{k}-N\right)
$$

where the first term represents mutual information of (36) and the second term corresponds to the energy constraint of (43). Also, $\alpha$ is the Lagrange multiplier. In order to optimize the 
coefficients $\gamma_{k}$, the following problem is solved [8]:

$$
\frac{\partial J}{\partial \gamma_{k}}=0, \text { subject to } \gamma_{k} \geq 0 .
$$

Then, the optimal coefficients $\gamma_{k}$ are given by

$$
\gamma_{k}=\max \left(\frac{1}{\alpha \phi_{k} \ln 2}-\frac{N_{0}}{\xi_{k} \sigma_{s}^{2}}, 0\right),
$$

which is calculated in the same manner as the classic waterfilling algorithm for the singular-value decomposition based multiple-input multiple-output scheme [19].

By normalizing (36) by the block duration of $N \tau T_{0}[3,4$, 7], the information rate of EVD-precoded FTN signaling with optimal power allocation is given by

$$
R=\frac{1}{N \tau T_{0}} \sum_{k=0}^{N-1} \log _{2}\left(1+\frac{\sigma_{s}^{2}}{N_{0}} \gamma_{k} \xi_{k}\right) \quad[\mathrm{bits} / \mathrm{sec}],
$$

where $\gamma_{k}$ is determined according to (46). ${ }^{1}$ Furthermore, by assuming an infinite block length, the associated capacity is given as follows [21]: ${ }^{2}$

$$
C_{\mathrm{opt}}^{\mathrm{FTN}}=\lim _{N \rightarrow \infty} \frac{1}{N \tau T_{0}} \sum_{k=0}^{N-1} \log _{2}\left(1+\frac{\sigma_{s}^{2}}{N_{0}} \gamma_{k} \xi_{k}\right) .
$$

In (48), it is assumed that the optimal coefficients $\gamma_{k}$ can be calculated for $N \rightarrow \infty$ and that the packing ratio is in the range of $\tau \geq 1 /(1+\beta)$.

\section{Detection Algorithm}

In this section, we present the detection algorithm for the proposed FTN signaling scheme with optimal power allocation. Let the weight matrix be $\mathbf{W}=\mathbf{U}^{H} \mathbf{D}^{H} \boldsymbol{\Lambda}^{-\frac{1}{2}} \mathbf{V}^{T}$, and then, the weighed received block $\mathbf{r}_{\mathrm{d}}$ is given by

$$
\begin{aligned}
\mathbf{r}_{\mathrm{d}}=\mathbf{W} \mathbf{y} & =\mathbf{U}^{H} \mathbf{D}^{H} \mathbf{D} \mathbf{U} \Gamma^{\frac{1}{2}} \mathbf{S}+\boldsymbol{\eta}_{w} \\
& =\boldsymbol{\Xi} \boldsymbol{\Gamma}^{\frac{1}{2}} \mathbf{S}+\boldsymbol{\eta}_{w},
\end{aligned}
$$

where we have $\boldsymbol{\eta}_{w}=\mathbf{W} \boldsymbol{\eta}$. Moreover, the correlation matrix of $\boldsymbol{\eta}_{w}$ is calculated by

$$
\begin{aligned}
\mathbb{E}\left[\boldsymbol{\eta}_{w} \boldsymbol{\eta}_{w}^{H}\right] & =\mathbf{W} \mathbb{E}\left[\boldsymbol{\eta} \boldsymbol{\eta}^{H}\right] \mathbf{W}^{H} \\
& =N_{0} \boldsymbol{\Xi} .
\end{aligned}
$$

As seen from (50) and (52), the received block is diagonalized and regarded as the $N$ independent parallel substreams, while the associated noise components are whitened. Hence, the received symbols are demodulated in a symbol-by-symbol manner.

The proposed FTN signaling transceiver has to calculate the matrix $\mathbf{D}^{H} \mathbf{D}$, the EVD of $\mathbf{D}^{H} \mathbf{D}$, the precoding matrix

\footnotetext{
${ }^{1}$ We describe the metric (47) as the information rate to distinguish from the rigorous capacity that assumes the infinite block length $(N \rightarrow \infty)$. We exploit (47) as the performance metric in Section III. To rigorously evaluate the achievable information rate for a given finite block size $N$, the penalty caused by the non-zero block error should be taken into account $[5,20]$.

${ }^{2}$ In [21], the coefficients similar to (46) were derived, while any analytical and numerical performance results were not provided.
}

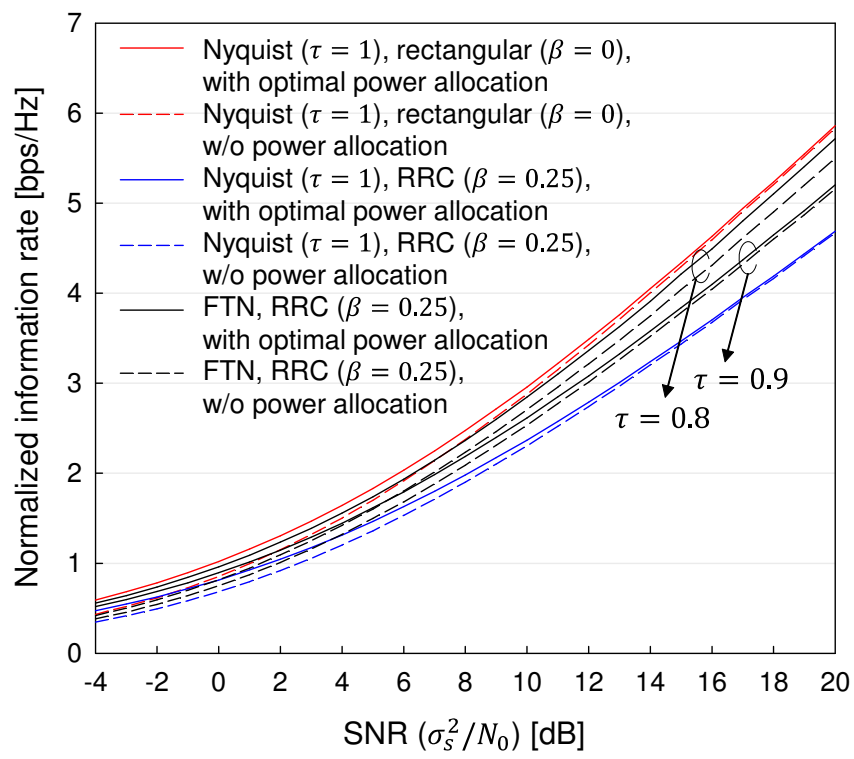

Fig. 1. Normalized information rate of the proposed FTN signaling scheme. The roll-off factor was set to $\beta=0.25$, while the symbol's packing ratio was given by $\tau=0.9$ and 0.8 . The block size and the delay spread were set to $N=1000$ and $L=20$, respectively.

$\mathbf{P}$, the weight matrix $\mathbf{W}$, and the optimized coefficients $\gamma_{k}{ }^{3}$ Furthermore, these calculations have to be updated in each coherence time. However, the aim of this paper is to determine the achievable performance upper bound of FTN signaling in a frequency-selective fading channel. To develop lowcomplexity detection of the proposed FTN signaling scheme is left for future studies.

\section{E. Implementable Packing Ratio Range}

As mentioned in Sections II-B, II-C, and II-D, the calculations of the inverse matrix $\mathbf{G}^{-1}=\mathbf{V} \boldsymbol{\Lambda}^{-1} \mathbf{V}^{T}$ are needed in the proposed FTN signaling scheme. The ISI matrix $\mathbf{G}$ is positive definite and its inverse exists for a finite block length $N[7,10]$. However, for $\tau<1 /(1+\beta)$, the part of the eigenvalues $\lambda_{k}$ become significantly low. Since it is a challenging task to precisely compute such a low eigenvalue in the standard floating-point arithmetic, truncation of substreams may have to be invoked in order to accurately implement the proposed FTN signaling scheme for a low symbol's packing ratio $\tau$ [8]. However, when $\tau \geq 1 /(1+\beta)$, the ISI matrix $\mathbf{G}$ does not include significantly low eigenvalues, and its inverse is accurately calculated [10]. To avoid this problem, we limit the packing ratio in the range of $1 /(1+\beta) \leq \tau \leq 1$ throughout the paper.

\section{INFORMATION RATE RESULTS}

Fig. 1 shows the information rate normalized by the associated bandwidth $2 W(1+\beta)$ for the proposed FTN signaling

\footnotetext{
${ }^{3}$ The inverse matrix $\mathbf{G}^{-1}$ is calculated offline, since the ISI matrix $\mathbf{G}$ is uniquely determined by $\tau$ and $\beta$ [8]
} 
scheme with and without power allocation. Here, in the proposed scheme without power allocation, the precoding matrix was set to $\mathbf{P}=\mathbf{U}$, i.e., $\boldsymbol{\Gamma}=\mathbf{I}_{N}$. The roll-off factor was set to $\beta=0.25$, while the symbol's packing ratio was set to $\tau=0.9$ and $0.8(=1 /(1+\beta))$. Moreover, we employed the block size of $N=1000$, and the delay spread was set to $L=20$. For comparison, we considered the Nyquistbased signaling ( $\tau=1)$ with and without power allocation, employing the RRC filter having $\beta=0.25$ and the idealistic rectangular shaping filter $(\beta=0)$ as the benchmarks. Observe in Fig. 1 that the proposed FTN signaling scheme with power allocation outperformed other benchmark schemes, employing the RRC shaping filter. Additionally, the proposed scheme with power allocation achieved the information rate close to the rectangular-filter bound $(\beta=0)$.

\section{Simulation Results}

We consider the channel-encoded system model of our proposed FTN signaling transceiver, employing the threestage serially-concatenated turbo coding architecture [22]. At the transmitter, information bits are encoded by the half-rate recursive systematic coding (RSC) encoder. The RSC-encoded bits are interleaved by the first interleaver $\Pi_{1}$. Then, the interleaved bits are encoded by the unity rate coding (URC) encoder [23], and the URC-encoded bits are interleaved by the second interleaver $\Pi_{2}$.

In the proposed scheme, the part of symbols are deactivated for maximizing the achievable information rate, according to (46). Furthermore, we introduce the concept of bit loading per symbol, where binary phase-shift keying (BPSK), quadrature PSK (QPSK), 16-quadrature amplitude modulation (QAM), 64-QAM, 256-QAM, and 1024-QAM schemes were assigned onto each activated symbols in order to achieve a target transmission rate [8]. The transmission rate $R_{t}$ of the proposed FTN signaling scheme is defined by

$$
R_{t}=\frac{1}{2} \cdot \frac{1}{2 W(1+\beta)} \cdot \frac{1}{N \tau T_{0}} \cdot \sum_{k=0}^{N-1} b_{k} \quad \text { bps } / \mathrm{Hz},
$$

where the coefficient $1 / 2$ corresponds to the rate loss due to the half-rate RSC encoder, while $b_{k}$ represents the number of bits assigned onto the $k$ th symbol.

The received sample block is decoded in an iterative manner between the three soft decoders. At the log-likelihood ratio (LLR) calculator, the LLR of each symbol is calculated based on the received sample and the extrinsic information fed back from the URC decoder. The LLR is easily calculated since the received sample is diagonalized and the associated noise is whitened. The number of outer iterations between the URC decoder and the RSC decoder is denoted by $I_{\text {out }}$, while the number of inner iterations between the URC decoder and the LLR calculator block is denoted by $I_{\mathrm{in}}$. We employed the halfrate RSC code with the constraint length of two and the octal generator polynomials of $(3,2)$. The number of outer iterations and that of inner iterations were set to $I_{\text {out }}=40$ and $I_{\text {in }}=2$, respectively. The block length was set to $N=1000$, while the
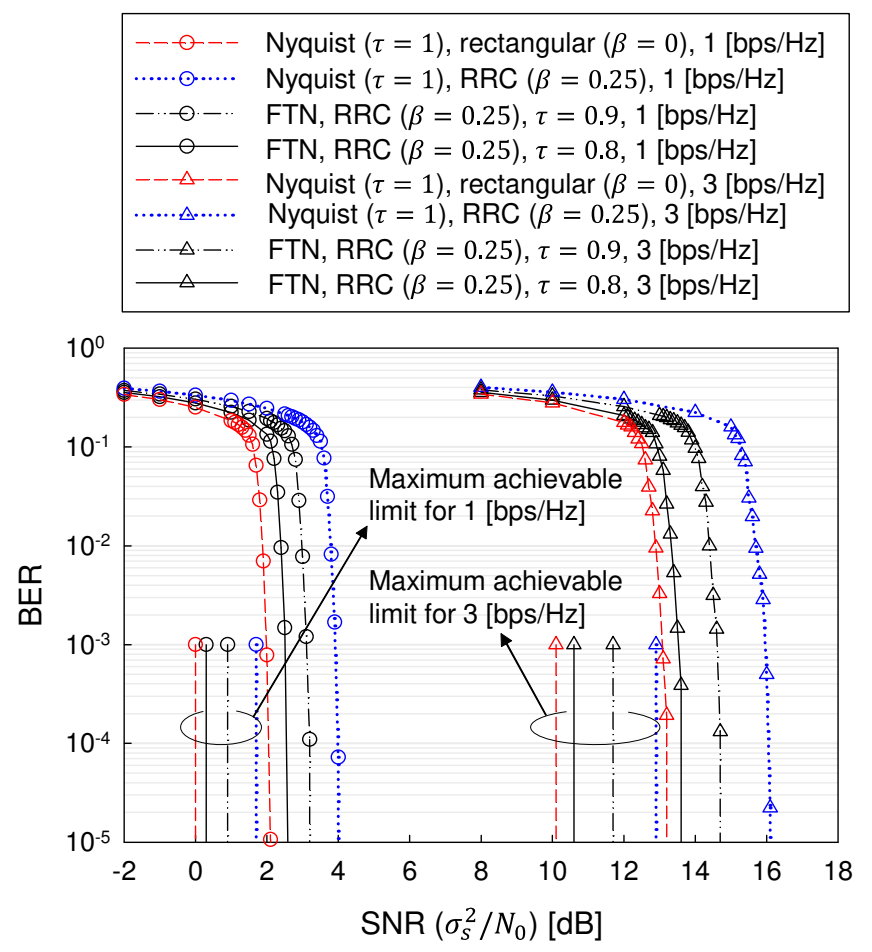

Fig. 2. BER performance of the proposed three-stage turbo-coded FTN signaling system. The roll-off factor was fixed to $\beta=0.25$, while the symbol's packing ratio was given by $\tau=1,0.9$, and 0.8 .

interleaver length was set to 200000 bits. ${ }^{4}$ We considered the target transmission rates of $R_{t}=1$ and $3 \mathrm{bits} / \mathrm{s} / \mathrm{Hz}$. The delay spread was given by $L=20$.

Fig. 2 shows the bit error ratio (BER) performance of the proposed FTN signaling scheme. The roll-off factor was fixed to $\beta=0.25$, while the symbol's packing ratio was given by $\tau=0.9$ and 0.8 . We plotted the BER performance of Nyquistbased signaling $(\tau=1)$ with the RRC filter having the rolloff factor $\beta=0.25$ and that of the idealistic rectangular filter $(\beta=0)$. Furthermore, the maximum achievable limit, corresponding to each BER curve, was plotted, which represents the bound calculated based on the information rate of (47). Observe in Fig. 2 that in each transmission rate scenario, the proposed FTN signaling with $\tau=0.8$ exhibited a better BER performance than that of $\tau=0.9$ and the Nyquist signaling benchmark with the same RRC filter. For the target rate of $3 \mathrm{bps} / \mathrm{Hz}$, the gap between the proposed FTN scheme with $\tau=0.8$ and the Nyquist signaling with the rectangular shaping filter was as low as $0.4 \mathrm{~dB}$.

Fig. 3 shows the power spectral density (PSD) of the proposed FTN signaling scheme with optimal power allocation, where the SNR of $10 \mathrm{~dB}$ and the delay spread of $L=20$ were considered. Moreover, the block length was set to $N=1000$, while sampling over the sufficiently-long

\footnotetext{
${ }^{4}$ In order to characterize the achievable performance bound, we ignored the effects of IBI similar to the previous studies of FTN signaling. The 200000 bits were divided into multiple blocks, and each block was independently precoded and transmitted.
} 


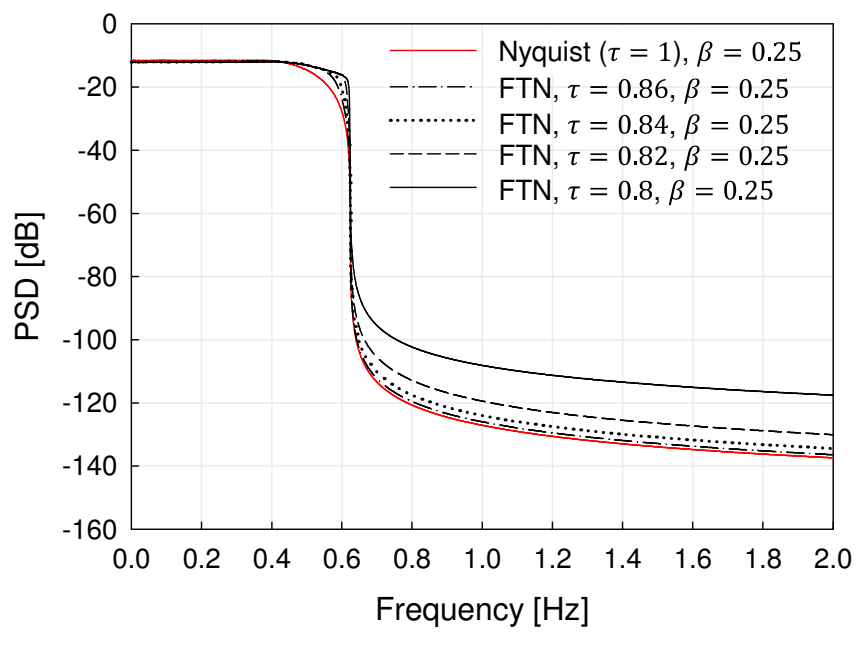

Fig. 3. PSD of the proposed FTN signaling scheme with optimal power allocation. The roll-off factor was fixed to $\beta=0.25$, while the packing ratio was given by $\tau=0.86,0.84,0.82$, and 0.8 . We considered the SNR of 10 $\mathrm{dB}$ and the delay spread of $L=20$.

time interval $\left[-100 T_{0}, N T_{0}+100 T_{0}\right]$. The roll-off factor was maintained to be $\beta=0.25$. The packing ratio was given by $\tau=0.86,0.84,0.82$, and 0.8. For comparison, the PSD of Nyquist-based signaling with optimal power allocation was also plotted. As shown in Fig. 3, the PSD of the proposed FTN scheme sharply dropped at the frequency of $W(1+\beta)=0.625$ $\mathrm{Hz}$, irrespective of the $\tau$ value. Although the spectral side-lobe level slightly increased upon decreasing $\tau$, it was sufficiently low in each $\tau$ scenario. Hence, the proposed FTN signaling scheme does not suffer from the effects of bandwidth broadening.

\section{CONCLUSIONS}

In this paper, we proposed the novel EVD-precoded FTN signaling scheme with optimal power allocation for a frequency-selective fading channel. We formulated mutual information of the proposed FTN signaling scheme, while optimizing the power allocation coefficients in terms of the derived mutual information. We showed that the proposed FTN signaling scheme achieved the information rate close to the bound of Nyquist signaling with the idealistic rectangular shaping filter. Moreover, our simulation results of the BER performance demonstrated that the proposed FTN signaling scheme with optimal power allocation outperforms the conventional FTN signaling scheme without power allocation and the classic Nyquist signaling scheme with the same RRC shaping filter, which is achieved without suffering from any bandwidth broadening.

\section{ACKNOWLEDGEMENT}

This work was supported in part by the Japan Society for the Promotion of Science (JSPS) KAKENHI (Grant Numbers 16KK0120, 17H03259, 17K18871, 20K22410), and in part by the Japan Science and Technology Agency (JST) Precursory
Research for Embryonic Science and Technology (PRESTO) (Grant Number JPMJPR1933).

\section{REFERENCES}

[1] J. E. Mazo, "Faster-than-Nyquist signaling," The Bell System Technical Journal, vol. 54, no. 8, pp. 1451-1462, Oct. 1975.

[2] J. Anderson, F. Rusek, and V. Öwall, "Faster-than-Nyquist signaling," Proceedings of the IEEE, vol. 101, no. 8, pp. 1817-1830, Aug. 2013.

[3] F. Rusek and J. B. Anderson, "Constrained capacities for faster-thanNyquist signaling," IEEE Transactions on Information Theory, vol. 55, no. 2, pp. 764-775, Feb. 2009.

[4] Y. G. Yoo and J. H. Cho, "Asymptotic optimality of binary faster-thanNyquist signaling," IEEE Communications Letters, vol. 14, no. 9, pp. 788-790, Sep. 2010.

[5] M. Mohammadkarimi, R. Schober, and V. W. S. Wong, "Channel coding rate for finite blocklength faster-than-Nyquist signaling," IEEE Communications Letters, vol. 25, no. 1, pp. 64-68, 2021.

[6] Y. J. D. Kim and J. Bajcsy, "Information rates of cyclostationary fasterthan-Nyquist signaling," in IEEE Canadian Workshop on Information Theory, May 2011.

[7] Y. J. D. Kim, "Properties of faster-than-Nyquist channel matrices and folded-spectrum, and their applications," in IEEE Wireless Communications and Networking Conference, Apr. 2016.

[8] T. Ishihara and S. Sugiura, "SVD-precoded faster-than-Nyquist signaling with optimal and truncated power allocation," IEEE Transactions on Wireless Communications, vol. 18, no. 12, pp. 5909-5923, Dec. 2019.

[9] K. Masaki, T. Ishihara, and S. Sugiura, "Tradeoff between calculation precision and information rate in eigendecomposition-based faster-thanNyquist signaling," IEEE Access, vol. 8, pp. 223 461-223 471, 2020.

[10] A. Gattami, E. Ringh, and J. Karlsson, "Time localization and capacity of faster-than-Nyquist signaling," in IEEE Global Communications Conference, San Diego, CA, USA, Dec. 2015, pp. 1-7.

[11] S. Sugiura, "Secrecy performance of eigendecomposition-based FTN signaling and NOFDM in quasi-static fading channel," TechRxiv, pp. 1-12, Mar. 2021, DOI:10.36227/techrxiv.14222033.v1.

[12] S. Sugiura, "Frequency-domain equalization of faster-than-Nyquist signaling," IEEE Wireless Communications Letters, vol. 2, no. 5, pp. 555558, Oct. 2013.

[13] S. Sugiura and L. Hanzo, "Frequency-domain-equalization-aided iterative detection of faster-than-Nyquist signaling," IEEE Transactions on Vehicular Technology, vol. 64, no. 5, pp. 2122-2128, May 2015.

[14] T. Ishihara and S. Sugiura, "Iterative frequency-domain joint channel estimation and data detection of faster-than-Nyquist signaling," IEEE Transactions on Wireless Communications, vol. 16, no. 9, pp. 62216231, Sep. 2017.

[15] Q. Shi, N. Wu, X. Ma, and H. Wang, "Frequency-domain joint channel estimation and decoding for faster-than-Nyquist signaling," IEEE Transactions on Communications, vol. 66, no. 2, pp. 781-795, Feb. 2018.

[16] A. Gattami and E. Ringh, "Pre-coding in a faster-than-Nyquist transmission system," Patent US 9838230B2, 2017.

[17] T. M. Cover and J. A. Thomas, Elements of Information Theory. New York: Wiley, 1991.

[18] A. Dembo, T. M. Cover, and J. A. Thomas, "Information theoretic inequalities," IEEE Transactions on Information Theory, vol. 37, no. 6, pp. 1501-1518, 1991.

[19] A. Goldsmith, Wireless communications. Cambridge University Press, 2005.

[20] Y. Polyanskiy, H. V. Poor, and S. Verdu, "Channel coding rate in the finite blocklength regime," IEEE Transactions on Information Theory, vol. 56, no. 5, pp. 2307-2359, 2010

[21] Y. J. D. Kim, "Faster than Nyquist transmission over continuous-time channels : Capacity analysis and coding," Ph.D. dissertation, McGill University, 2013.

[22] S. Sugiura, S. Chen, and L. Hanzo, "Coherent and differential spacetime shift keying: A dispersion matrix approach," IEEE Transactions on Communications, vol. 58, no. 11, pp. 3219-3230, Nov. 2010.

[23] D. Divsalar, S. Dolinar, and F. Pollara, "Serial concatenated trellis coded modulation with rate-1 inner code," in IEEE Global Telecommunications Conference, vol. 2, San Francisco, CA, Nov. 2000, pp. 777-782. 